\title{
Finite-Size Scaling Analysis of Generalized Mean-Field Theories
}

\author{
Steffen D. Frischat* and Reimer Kühn ${ }^{\dagger}$ \\ Institut für Theoretische Physik \\ Universität Heidelberg \\ Philosophenweg 19 \\ 69120 Heidelberg, Germany
}

\begin{abstract}
We investigate families of generalized mean-field theories that can be formulated using the Peierls-Bogoliubov inequality. For test-Hamiltonians describing mutually non-interacting subsystems of increasing size, the thermodynamics of these mean-field type systems approaches that of the infinite, fully interacting system except in the immediate vicinity of their respective mean-field critical points. Finite-size scaling analysis of this mean-field critical behaviour allows to extract the critical exponents of the fully interacting system. It turns out that this procedure amounts to the coherent anomaly method (CAM) proposed by Suzuki, which is thus given a transparent interpretation in terms of conventional renormalization group ideas. Moreover, given the geometry of approximating systems, we can identify the family of approximants which is optimal in the sense of the Peierls-Bogoliubov inequality. In the case of the 2- $d$ Ising model it turns out that, surprisingly, this optimal family gives rise to a spurious singularity of thermodynamic functions.
\end{abstract}

${ }^{*}$ Present address: Max-Planck-Institut für Kernphysik, Postfach 103980, 69029 Heidelberg, FRG

${ }^{\dagger}$ Supported by a Heisenberg fellowship 


\section{Introduction}

Standard wisdom has it that closed form approximations and renormalization group methods play complementary roles in the analysis of thermodynamic behaviour of many-particle systems. The former usually generate mean-field type theories, and as such often provide efficient tools to obtain a good qualitative picture of a given system's thermodynamics. Equations of state and qualitatively correct phase diagrams are relatively easily calculated. Famous examples of such approaches are the van der Waals theory of imperfect gases and the Weiss self-consistent theory of ferromagnetism. With respect to a quantitative description of phase transitions, however, these theories invariably fail and produce wrong critical exponents. Renormalization group ideas, on the other hand, provide a satisfactory theoretical description of critical phenomena and the interplay of critical exponents. Except for the determination of critical exponents, though, renormalization group calculations are rather involved and do not easily allow to obtain a picture of the system's thermodynamic properties.

In the course of time, various refinements of the standard mean-field theory have been proposed; for an overview, see [1]. Patterned after the first such attempt due to Bethe [2], short range correlations of the dynamic variables are taken into account by considering small clusters. Equations of state are generated in the form of self-consistency equations which impose certain physically plausible constraints, such as homogenity of the order parameter. This inclusion of short range correlations, hence of additional phase space, leads to improved (i. e., lower) estimates of the critical temperature. But it fails to produce improved critical exponents - the reason being that critical phenomena are dominated by long-range correlations.

An alternative, more systematic construction of mean-field type theories derives from a variational scheme based on the Peierls-Bogoliubov (PB) inequality [3]. This inequality is based on convexity arguments and states that, given a system with Hamiltonian $\mathcal{H}$, its free energy $F$ can be approximated from above by the trial "free energy" $\Phi$ as

$$
F \leq \Phi:=F_{0}+\left\langle\mathcal{H}-\mathcal{H}_{0}\right\rangle_{0}=\langle\mathcal{H}\rangle_{0}-T S_{0}
$$

Here, $\mathcal{H}_{0}$ is an arbitrary test-Hamiltonian for the system in question, which depends on some set $\left\{h_{\alpha}\right\}$ of variational parameters. $\langle\ldots\rangle_{0}$ denotes the average over the Gibbs distribution generated by $\mathcal{H}_{0}$, and $F_{0}$ and $S_{0}$ denote the corresponding free energy and entropy. The idea 
is to choose $\mathcal{H}_{0}$ such that the corresponding Gibbs distribution is analytically or numerically tractable and to determine the variational parameters $h_{\alpha}$ so as to minimize the right hand side of (1.1). The resulting minimization conditions replace the above self-consistency equations and generate the system's equations of state. To state an example, let $\mathcal{H}$ describe the Ising spin system on a lattice in $d$ space dimensions. The simplest approximating $\mathcal{H}_{0}$ then describes a system of non-interacting spins in a mean field $h_{0}$, that is, $\mathcal{H}_{0}=-h_{0} \sum_{i} S_{i}$. Minimizing the corresponding trial free energy with respect to $h_{0}$ generates the conventional Weiss mean-field equation of state. For a recent application of this method to $\mathrm{CsNiF}_{3}$ chains, see 沟.

Short range correlations can now be taken into account by choosing a system of mutually independent clusters of spins, which together make up the whole system. Increasing the size of these clusters, one obtains a scheme of approximations that should systematically approach the thermodynamics of the underlying, fully interacting (spin) system.

Despite the fact that every PB system of finite (or quasi one-dimensional) geometry exhibits mean-field type critical behaviour, the true critical exponents of the underlying system can be extracted by invoking finite-size scaling (FSS) ideas. Thermodynamic functions can be evaluated as functions of cluster size. We will show that this procedure is equivalent to Suzuki's coherent anomaly method (CAM) [5, 6]. CAM is thus demonstrated to be firmly rooted in the FSS philosophy and hence in conventional renormalization group ideas.

Within the general PB scheme, and for a given cluster geometry, various families of approximating systems can be constructed which differ in number, symmetries, or even nature of their variational parameters. Of all of those, the optimal family - in the sense of minimal trial free energy - is the one with the largest set of independent variational parameters compatible with the symmetries of the system.

In the present paper, we will explore a collection of approximating sequences for the $2-d$ Ising model. Two families will prove to be of special interest: cyclically "closed" strips, that display Suzuki's coherent anomaly, and "open" strips of lower symmetry, that can be identified as the optimal PB sequence. Surprisingly, this optimal family gives rise to a spurious singularity of thermodynamic functions making any extrapolation to the full $2-d$ model based on the open strip's mean-field critical behaviour impossible. In this restricted sense, the complementarity between closed form approximations and the RG reappears. 
The outline of our paper is as follows. In Sec. 2, we introduce a collection of variational trial systems for the 2- $d$ Ising system, based on the Peierls-Bogoliubov inequality. Test-Hamiltonians will be defined on $M \times \infty$ strips which may be open or closed in the $M$ direction. We show that, quite generally, all but one of the extremization conditions for the variational parameters can be solved explicitly, leaving only one non-trivial condition as the equation of state. In Sec. 3, we use finite-size scaling to derive the scaling of the mean-field critical temperatures $T_{M}$ with strip width $M$. For test-ensemles based on cyclically closed strips (Sec. 3.1), this provides a first method to extract the susceptibility exponent $\gamma$ of the underlying, fully interacting system. The second method uses the mean-field susceptibilities and their scaling with strip width $M$. The coherent anomaly, which is at the base of this method, is given a simple explanation as a standard FSS phenomenon. Sec 3.2 is devoted to an analysis of variational approximants defined on open strips. These were identified in Sec. 2 as the best sequence of variational approximants of strip-geometry in the framework of the PB inequality. Contrary to expectations, a FSS analysis of these "optimal" variational approximants predicts a spurious singularity of thermodynamic functions that precludes any extrapolation attempt to the two-dimensional system. Sec. 4 is a discussion of the results.

\section{Variational approximants for the 2-d Ising model}

We now introduce a collection of approximating systems for the 2- $d$ Ising model, based on the Peierls-Bogoliubov inequality. Let

$$
\mathcal{H}=-J \sum_{(i j)} s_{i} s_{j}-H \sum_{i} s_{i}
$$

describe the fully interacting system on a square lattice of $N^{\prime} \times N$ Ising spins, and let us assume periodic boundary conditions in both directions. As a test-ensemble we use a system of mutually non-interacting strips of size $M \times N$ with periodic boundary conditions in the "longitudinal" $N$ direction, and either free or periodic boundary conditions in the "transverse" $M$ direction. 


\subsection{Test-ensembles based on closed strips}

Let us first consider the version which is cyclically closed in both directions. A simple Hamiltonian for a single strip of width $M$, which exhibits full translational invariance in both directions, is given by

$$
\mathcal{H}_{M, N}^{c}=-h_{T} \sum_{(i j)_{T}} s_{i} s_{j}-h_{L} \sum_{(i j)_{L}} s_{i} s_{j}-(H+h) \sum_{i} s_{i}
$$

where we have introduced three variational parameters $\left\{h_{\alpha}\right\}:=\left\{h_{T}, h_{L}, h\right\}$ a coupling $h_{T}$ for transverse nearest neighbours $(i j)_{T}$, a coupling $h_{L}$ for longitudinal nearest neighbours $(i j)_{L}$, and a variational field $h$. The trivial effect of the external field $H$ has been absorbed into the definition of $h$.

Let us denote the free energy density of an isolated strip with Hamiltonian (2.2) by $f_{M}^{c}=$ $f_{M}^{c}\left(T, H,\left\{h_{\alpha}\right\}\right)$. Assuming $N^{\prime}$ to be an integer multiple of $M$, the PB inequality for this setup states that

$$
\begin{aligned}
F & \leq \Phi_{M}^{c}\left(T, H,\left\{h_{\alpha}\right\}\right)=: N^{\prime} N \phi_{M}^{c}\left(T, H,\left\{h_{\alpha}\right\}\right) \\
& =N^{\prime} N\left\{f_{M}^{c}+\left(J\left(1-\frac{1}{M}\right)-h_{T}\right) \frac{\partial f_{M}^{c}}{\partial h_{T}}+\left(J-h_{L}\right) \frac{\partial f_{M}^{c}}{\partial h_{L}}-\left(J \frac{1}{M} \frac{\partial f_{M}^{c}}{\partial h}+h\right) \frac{\partial f_{M}^{c}}{\partial h}\right\}
\end{aligned}
$$

Here we have used the fact that $\left\langle s_{i} s_{j}\right\rangle_{0}$ is given by $\left(-\partial f_{M}^{c} / \partial h_{T}\right)$ for transverse and by $\left(-\partial f_{M}^{c} / \partial h_{L}\right)$ for longitudinal nearest neighbours within a strip, while $\left\langle s_{i} s_{j}\right\rangle_{0}=\left\langle s_{i}\right\rangle_{0}\left\langle s_{j}\right\rangle_{0}=\left(-\partial f_{M}^{c} / \partial h\right)^{2}=$ : $\left(m_{M}^{c}\right)^{2}$ for spins belonging to different strips. Here $m_{M}^{c}$ denotes the magnetization of a strip of width $M$.

From (2.3) the minimization conditions are obtained in the form

$$
\varphi_{\alpha}:=\frac{\partial \phi_{M}^{c}}{\partial h_{\alpha}}=\sum_{\beta} \frac{\partial^{2} f_{M}^{c}}{\partial h_{\alpha} \partial h_{\beta}} \psi_{\beta}=0
$$

with $h_{\alpha} \in\left\{h_{T}, h_{L}, h\right\}$, and

$$
\left\{\psi_{\beta}\right\}=\left\{J\left(1-\frac{1}{M}\right)-h_{T}, J-h_{L}, J \frac{2}{M} m_{M}^{c}-h\right\} .
$$

Due to the concavity of $f_{M}^{c}$ as a function of the $h_{\alpha}$ - the Hessian $\left(\partial^{2} f_{M}^{c} / \partial h_{\alpha} \partial h_{\beta}\right)_{\alpha, \beta}$ is a strictly negative definite matrix - the solution of (2.4) can be read off immediately: it is $\psi_{\beta}=0$, i. e.,

$$
h_{T}=J\left(1-\frac{1}{M}\right)
$$




$$
\begin{aligned}
h_{L} & =J \\
h & =J \frac{2}{M} m_{M}^{c}=-J \frac{2}{M} \frac{\partial f_{M}^{c}}{\partial h} .
\end{aligned}
$$

Of these, only the last one, (2.8), is a non-trivial transcendental equation with a solution that varies with temperature $T$ and external field $H$. It determines the mean field $h=h(T, H)$. The variational (mean-field) free energy density computed within this approach is then

$$
f_{M}^{m f}(T, H)=\phi_{M}^{c}\left(T, H, h_{T}, h_{L}, h(T, H)\right)
$$

with variational parameters $\left\{h_{\alpha}\right\}$ determined by (2.6) $-(2.8)$.

Thermodynamic functions are obtained by differentiation of $f_{M}^{m f}(T, H)$ along the solution manifold given by (2.8). This yields

$$
m_{M}^{m f}=-\left(\frac{d f_{M}^{m f}(T, H)}{d H}\right)_{\varphi, T}=-\frac{\partial \phi_{M}^{c}}{\partial H}-\sum_{\alpha} \frac{\partial \phi_{M}^{c}}{\partial h_{\alpha}}\left(\frac{d h_{\alpha}}{d H}\right)_{\varphi, T},
$$

where the subscripts $\varphi, T$ denote differentiation along the manifold $\varphi_{\alpha}=0$ at constant $T$. Partial differentials are taken as usual. With the help of (2.9) and $\partial \phi_{M}^{c} / \partial h_{\alpha} \equiv 0$ one obtains the mean-field magnetization

$$
m_{M}^{m f}=-\frac{\partial f_{M}^{c}}{\partial H}
$$

In a similar vein, the mean-field susceptibility is found to be

$$
\begin{aligned}
\chi_{M}^{m f} & =\left(\frac{d m_{M}^{m f}(T, H)}{d H}\right)_{\varphi, T}=\frac{\partial m_{M}^{m f}}{\partial H}+\sum_{\alpha} \frac{\partial m_{M}^{m f}}{\partial h_{\alpha}}\left(\frac{d h_{\alpha}}{d H}\right)_{\varphi, T} \\
& =-\frac{\partial^{2} f_{M}^{c}}{\partial H^{2}}+J \frac{2}{M} \frac{\left(\partial^{2} f_{M}^{c} / \partial H \partial h\right)^{2}}{1+J \frac{2}{M} \partial^{2} f_{M}^{c} / \partial h^{2}} .
\end{aligned}
$$

That is, magnetization and mean-field susceptibility can be expressed in terms of free partial derivatives of the strip free energy $f_{M}^{c}$ of a strip; the strip being described by the Hamiltonian $\mathcal{H}_{M N}^{c}$ evaluated at parameter values given by (2.6) - (2.8).

In principle, one may try to improve the approximation by introducing additional variational parameters that represent "generalized" couplings beyond the ones already contained in (2.2) which generate interaction terms added to $\mathcal{H}_{M N}^{c}$ in a translationally invariant way. To be specific, we modify $\mathcal{H}_{M, N}^{c}$ according to

$$
\mathcal{H}_{M, N}^{c} \longrightarrow \mathcal{H}_{M, N}^{c}-\sum_{\omega \subseteq \Omega} h_{\omega} \sum_{i}\left(\prod_{j \in \omega} s_{j+i}\right)
$$


where $\Omega$ denotes a collection of subsets of the $M \times N$ strip which are mutually non-equivalent under translation. It turns out that such an enlarged space of variational parameters does not actually improve the variational free energy, because the enlarged set of minimization conditions is solved by (2.6) $-(2.8)$ and $h_{\omega}=0$ for all of the added $\omega \subseteq \Omega$. To see this, note that the modification (2.13) implies a corresponding replacement

$$
\left\langle\mathcal{H}_{0}\right\rangle_{0} \longrightarrow\left\langle\mathcal{H}_{0}\right\rangle_{0}+N N^{\prime} \sum_{\omega \subseteq \Omega} h_{\omega} \frac{\partial f_{M}^{c}}{\partial h_{\omega}},
$$

where $f_{M}^{c}$ is now the free energy corresponding to the modified Hamiltonian (2.13). Hence the enlarged set of minimization conditions can be formulated in complete analogy to (2.4), albeit with an enlarged set of variational parameters, $h_{\alpha} \in\left\{h_{T}, h_{L}, h,\left\{h_{\omega}\right\}_{\omega \subseteq \Omega}\right\}$ and

$$
\left\{\psi_{\beta}\right\}=\left\{J\left(1-\frac{1}{M}\right)-h_{T}, J-h_{L}, J \frac{2}{M} m_{M}^{c}-h,\left\{-h_{\omega}\right\}_{\omega \subseteq \Omega}\right\} .
$$

Due to the concavity of $f_{M}^{c}\left(T, H,\left\{h_{\alpha}\right\}\right)$ the assertion follows, that is $h_{\omega}=0$ for all $\omega \subseteq \Omega$.

\subsection{Test-ensembles based on open strips}

An alternative sequence of test systems is defined by considering "open" $M \times N$ strips with free boundary conditions in the transverse $M$ direction. While such strips retain the full translational invariance in the closed $N$ direction, they exhibit only a reflection symmetry $j \rightarrow M+1-j$ in the open $M$ direction. This reduced symmetry group allows to introduce a considerably larger set of independent variational parameters. A simple Hamiltonian respecting these symmetries is given by

$$
\begin{aligned}
\mathcal{H}_{M N}^{o}=-\sum_{i=1}^{N} & \left\{\sum_{j=1}^{\mu} h_{T, j} \sum_{\kappa \in\{j, M-j\}} s_{i, \kappa} s_{i, \kappa+1}+\sum_{j=1}^{\mu^{\prime}} h_{L, j} \sum_{\kappa \in\{j, M+1-j\}} s_{i, \kappa} s_{i+1, \kappa}\right. \\
& \left.+\sum_{j=1}^{\mu^{\prime}}\left(H+h_{j}\right) \sum_{\kappa \in\{j, M+1-j\}} s_{i, \kappa}\right\},
\end{aligned}
$$

where $\mu=[M / 2]$ and $\mu^{\prime}=[(M+1) / 2]$ with the convention that $[k]$ denotes the largest integer less than or equal to $k$. Also, we have introduced a two dimensional notation to label the vertices of the strip. Note that the variational fields and couplings vary from row to row, but respect the reflection invariance of the open strip in the $M$ direction. The total number of independent variational parameters is $3 M / 2$ for even $M$, and $(3 M+1) / 2$ for odd $M$. 
Denoting by $f_{M}^{o}=f_{M}^{o}\left(T, H,\left\{h_{T, j}\right\},\left\{h_{L, j}\right\},\left\{h_{j}\right\}\right)$ the free energy density of an isolated (open) strip with Hamiltonian (2.16), and assuming $N^{\prime}$ to be an integer multiple of $M$, we conclude by the Peierls-Bogoliubov inequality that

$$
\begin{aligned}
F \leq & \Phi_{M}^{o}\left(T, H,\left\{h_{T, j}\right\},\left\{h_{L, j}\right\},\left\{h_{j}\right\}\right) \\
= & N^{\prime} N\left\{f_{M}^{o}+\sum_{j=1}^{\mu}\left(J-h_{T, j}\right) M \frac{\partial f_{M}^{o}}{\partial h_{T, j}}+\sum_{j=1}^{\mu^{\prime}}\left(J-h_{L, j}\right) M \frac{\partial f_{M}^{o}}{\partial h_{L, j}}\right. \\
& \left.-\sum_{j=2}^{\mu^{\prime}} h_{j} M \frac{\partial f_{M}^{o}}{\partial h_{j}}-\left(J \frac{M}{2} \frac{\partial f_{M}^{o}}{\partial h_{1}}+h_{1}\right) M \frac{\partial f_{M}^{o}}{\partial h_{1}}\right\} .
\end{aligned}
$$

The minimization conditions are formally the same as (2.4), with $f_{M}^{c}$ replaced by $f_{M}^{o}$, with $h_{\alpha} \in\left\{\left\{h_{T, j}\right\},\left\{h_{L, j}\right\},\left\{h_{j}\right\}\right\}$, and

$$
\left\{\psi_{\beta}\right\}=\left\{\left\{J-h_{T, j}\right\},\left\{J-h_{L, j}\right\},\left\{-h_{j}\right\}_{j=2, . ., \mu^{\prime}}, J m_{M, 1}^{o}-h_{1}\right\} .
$$

Here $m_{M, 1}^{o}=\left\langle s_{i, 1}\right\rangle_{o}=-(M / 2) \partial f_{M}^{o} / \partial h_{1}$. Again, due to concavity, the solution of the minimization conditions are $\psi_{\beta}=0$, or

$$
\begin{aligned}
h_{T, j} & =J, \\
h_{L, j} & =J, \\
h_{j} & =0 \quad \text { for } \quad j=2, \ldots, \mu^{\prime}, \\
h_{1} & =J m_{M, 1}^{o}=-J \frac{M}{2} \frac{\partial f_{M}^{o}}{\partial h_{1}} .
\end{aligned}
$$

That is, all variational couplings are equal to the coupling $J$ of the underlying system, and all variational fields, except for the boundary field $h_{1}$, vanish.

Thermodynamic functions are obtained as before. In particular, the mean-field-magnetization of an "open" strip of width $M$ is given by

$$
m_{M}^{m f}=-\frac{\partial f_{M}^{o}}{\partial H}
$$

and the mean-field susceptibility by

$$
\chi_{M}^{m f}=-\frac{\partial^{2} f_{M}^{o}}{\partial H^{2}}+J \frac{M}{2} \frac{\left(\partial^{2} f_{M}^{o} / \partial H \partial h_{1}\right)^{2}}{1+J \frac{M}{2} \partial^{2} f_{M}^{o} / \partial h_{1}^{2}} .
$$

As above, thermodynamic functions can be expressed in terms of free partial derivatives of the free energy of a single strip of corresponding geometry described by the Hamiltonian (2.16) with parameter values given by 2.19) - 2.22). 
As in the case of closed approximants, any attempt to enlarge the space of variational parameters by adding further (multi-spin) interactions to $\mathcal{H}_{M N}^{o}$ does not lead to any improvement of the variational approximations: the minimization condition requires the corresponding coupling constants to be zero. In particular, an extra variational coupling $h_{T, M}$ which would close the strip in the transverse $M$ direction will have to vanish, rendering the strip open again at optimally chosen variational parameters. Therefore, within the framework of strip geometries, the testensemble based on open strips with Hamiltonian (2.16) may be identified as optimal in the sense of the PB inequality. It uses the largest meaningful set of independent variational parameters compatible with the lowest symmetry of $M \times \infty$ strips. Hence, the minimum obtained by $f_{M}^{o}$ is the total minimum of sensible trial free energies $\Phi$.

\section{Finite-size scaling analysis of variational approximants}

We now turn to an evaluation of thermodynamic functions computed within the variational approximation schemes described in Sec. 2. The dependence of the thermodynamic behaviour of mean-field test-strips on their width $M$ will be extracted by the use of finite-size scaling. Wherever possible, we will determine critical exponents.

In both cases, only one variational parameter turned out to be non-trivial. In the case of test-ensembles living on closed strips, this parameter is a variational field $h$ acting homogeneously on all spins and determined by (2.8),

$$
h=J \frac{2}{M} m_{M}^{c}
$$

whereas in the case of test-ensembles based on open strips, it is a boundary field $h_{1}$ acting only on the first and the $M$-th row of each strip, and which is determined by (2.22),

$$
h_{1}=J m_{M, 1}^{o}
$$

In both versions of the variational scheme, the approximation $T_{M}$ of the critical point $T_{c}$ is signalled by the appearance of non-zero solutions of the variational field, $h$ or $h_{1}$, respectively. As the temperature $T$ is lowered, the bifurcation from the zero solution occurs when (setting $J=1)$

$$
1=\frac{2}{M} \frac{\partial m_{M}^{c}}{\partial h}(T, H=0, h=0)
$$


in the "closed" variant, and when

$$
1=\frac{\partial m_{M, 1}^{o}}{\partial h_{1}}\left(T, H=0, h_{1}=0\right)
$$

in the "open" variant. The solutions of these equations define the mean-field critical temperatures $T_{M}$. In the following, we discuss the scaling analysis of these equations and of the corresponding divergence of the mean-field susceptibilities (2.12) and (2.24). The two different setups will be considered seperately.

\subsection{Finite-size analysis of test-ensembles based on closed strips}

Let us begin with the sequence of approximants based on closed homogeneously magnetized strips. In this setup both the variational field $h$ and the external field $H$ act homogeneously on all spins. As we have chosen $\mathcal{H}_{M N}^{c}$ to depend on these fields through their sum $H+h$, the free energy $f_{M}^{c}$, is a function of $(H+h)$ only, and we can replace partial derivatives of $f_{M}^{c}$ with respect to $h$ by partial derivatives with respect to $H$ and vice versa. Denoting the "free" susceptibility of a closed strip of circumference $M$ by

$$
\chi_{M}^{c}(T, h)=-\frac{\partial^{2} f_{M}^{c}}{\partial H^{2}}(T, H=0, h),
$$

we can reformulate the critical condition (3.1) as

$$
1=\frac{2}{M} \chi_{M}^{c}\left(T_{M}, 0\right)
$$

and the expression (2.12) for the mean-field susceptibility according to

$$
\begin{aligned}
\chi_{M}^{m f}(T, H=0) & =\chi_{M}^{c}(T, h)+\frac{2}{M} \frac{\left(\chi_{M}^{c}(T, h)\right)^{2}}{1-\frac{2}{M} \chi_{M}^{c}(T, h)} \\
& =\frac{\chi_{M}^{c}(T, h)}{1-\frac{2}{M} \chi_{M}^{c}(T, h)} .
\end{aligned}
$$

These two expressions are now directly amenable to analysis by FSS [7].

Analysis of (3.4) will give the asymptotic behaviour of the reduced mean-field critical temperatures $t_{M}:=\left(T_{M}-T_{c}\right) / T_{M}$. Let us first recall the finite-size behaviour of the free energy of an Ising strip of width $M$ in zero field. Close to the critical temperature $T_{c}$ of the two dimensional Ising system, the singular part of its zero field susceptibility is given by the finite-size scaling expression

$$
\chi_{M}(T) \sim \chi_{\infty}(T) \mathcal{Q}\left(\frac{\xi_{\infty}(T)}{M}\right) \sim|t|^{-\gamma} \mathcal{Q}_{h o m}\left(\frac{|t|^{-\nu}}{M}\right),
$$


where $t=\left(T-T_{c}\right) / T_{c}$. This expression holds for open and closed strips alike, albeit with different scaling functions. The behaviour of the scaling function $\mathcal{Q}_{\text {hom }}(z)$ in the limits $z:=$ $\left(|t|^{-\nu} / M\right) \rightarrow 0$ and $z \rightarrow \infty$ can be easily determined. These limits correspond to the cases $M \rightarrow \infty$ at non-critical temperature $T \neq T_{c}$, or $|t| \rightarrow 0$ at finite size $M<\infty$, respectively. Regularity of the left hand side of (3.6) in these cases implies the power laws $\mathcal{Q}_{\text {hom }}(z) \sim 1$ for $z \rightarrow 0$ and $\mathcal{Q}_{\text {hom }}(z) \sim z^{-\gamma / \nu}$ for $z \rightarrow \infty$.

This can be applied to the strips considered above. At temperatures above and at the mean-field critical temperature $T_{M}$, no variational field is present. Under the assumption that the temperatures $T_{M}$ are sufficiently close to $T_{c}$ for large $M$, standard finite-size scaling holds, and (3.4) becomes

$$
1 \sim \frac{t_{M}^{-\gamma}}{M} \mathcal{Q}_{h o m}\left(\frac{t_{M}^{-\nu}}{M}\right)
$$

As $M \rightarrow \infty$, the argument $z_{M}:=\left(t_{M}{ }^{-\nu} / M\right)$ of the scaling function at $T=T_{M}$ can either vanish, converge to a non-zero constant, or diverge. The latter two cases lead to contradictions $(\gamma \neq \nu$ assumed), leaving $z_{M} \rightarrow 0$ as the only possibility. Hence $\mathcal{Q}_{h o m}\left(z_{M}\right) \sim 1$ as $M \rightarrow \infty$, and by (3.7) the mean-field critical temperatures $T_{M}$ asymptotically scale as

$$
t_{M}=\left(T_{M}-T_{c}\right) / T_{c} \sim M^{-1 / \gamma}
$$

Note that $\gamma$ is the true susceptibility exponent of the underlying 2- $d$ Ising model so that (3.8) can be used to determine both $T_{c}$ and $\gamma$.

The same analysis, applied to (3.5), gives the behaviour of the mean-field susceptibility $\chi_{M}^{m f}$ in the vicinity of the mean-field critical temperatures $T_{M}$. Expanding the denominator in small temperature differences $t-t_{M}=\left(T-T_{M}\right) / T_{c}$ above $T_{M}$ gives

$$
1-\frac{2}{M} t^{-\gamma} \mathcal{Q}_{h o m}(z) \simeq\left(t-t_{M}\right) \frac{2}{M} t_{M}{ }^{-\gamma-1}\left[\gamma \mathcal{Q}_{h o m}\left(z_{M}\right)+\nu z_{M} \mathcal{Q}_{h o m}^{\prime}\left(z_{M}\right)\right]
$$

The second term in the square brackets can be neglected, as $z_{M} \rightarrow 0$ and $\mathcal{Q}_{\text {hom }}^{\prime} \rightarrow 0$ for $M \rightarrow \infty$. We substitute $M \propto t_{M}{ }^{-\gamma}$, cancel $\chi_{M}^{c}\left(T_{M}, 0\right) \propto t_{M}{ }^{-\gamma} \mathcal{Q}_{h o m}\left(z_{M}\right)$, and finally arrive at

$$
\chi_{M}^{m f}(T) \propto \frac{1}{\left(t-t_{M}\right)} \frac{1}{t_{M}^{\gamma-1}} .
$$

Eq. (3.10) exhibits the usual mean-field divergence of the susceptibility $\chi_{M}^{m f}(T) \propto\left(t-t_{M}\right)^{-1}$ as $t \rightarrow t_{M}$. Note that the prefactor $t_{M}{ }^{-(\gamma-1)}$ itself diverges as $M \rightarrow \infty$, a phenomenon for which 
Suzuki coined the term coherent anomaly [5]. Obviously, the coherent anomaly provides a second possibility to extract the asymptotic susceptibility exponent $\gamma$ of the underlying system from the sequence of mean-field approximations. Our considerations clearly show that this method is entirely rooted in the FSS philosophy, hence in conventional renormalization group ideas. This relationship has hitherto been much less clear in the literature.

The dependence of the mean-field critical temperatures $T_{M}$ on the strip width $M$ is displayed in Fig. 1. The convergence to the asymptotic value is fairly slow, as can be anticipated from (3.8). Nevertheless, good extrapolation algorithms (see, for instance, [13) predict $T_{M} \rightarrow T_{\infty}=$ $2.26 \pm 0.01$, which is reasonably close to the exact value of $T_{c} \simeq 2.269$. Setting $T_{c}=T_{\infty}$ in (3.8), we obtain an estimate for $\gamma$, viz., $\gamma \rightarrow 1.77 \pm 0.03$ as $M \rightarrow \infty$. While not extraordinary, this result is also not too far off the mark.

Fig. 2 shows the values of $\gamma$, obtained from the ratio of prefactors $\bar{\chi}_{M}=t_{M}{ }^{-(\gamma-1)}$ of the mean-field susceptibility (3.10) for two successive strip widths $M$ and $M^{\prime}=M+1$. Assuming $T_{c}=T_{\infty}$, we extrapolate this sequence of $\gamma$ values to $\gamma_{\infty}=1.765 \pm 0.01$, which produces a reasonably good agreement with the exact result $\gamma=1.75$ for the susceptibility exponent. With the exact value for $T_{c}$, the extrapolation yields a slightly better result, $\gamma_{\infty}=1.751 \pm 0.01$, which gives the susceptibility exponent to within less than $1 \%$ of the exact result.

\subsection{Finite-size analysis of test-ensembles based on open strips}

Much to our surprise, the approximation scheme breaks down in the case of the ensemble of open, inhomogeneously magnetized strips - the family of systems we identified as ideal in the sense of the Peierls-Bogoliubov inequality!

By concavity, we have singled out the boundary field $h_{1}$ as the only non-trivial variational parameter. It affects only two spins per column. The self-consistency equation is given by (2.22), and the critical condition by

$$
1=\frac{\partial m_{M, 1}^{o}}{\partial h_{1}}\left(T_{M}, 0,0\right)
$$

Numerical values of $T_{M}$, obtained by transfer matrix calculations are plotted in Fig. 3. Unexpectedly, as $M \rightarrow \infty$, they converge to a temperature $T_{\infty} \simeq 2.64$ which is different from the correct critical temperature $T_{c} \simeq 2.27$ of the two-dimensional Ising model. 
For a qualitative explanation of this behaviour, we again refer to FSS analysis. We note that the susceptibility

$$
\frac{\partial m_{M, 1}^{o}}{\partial h_{1}}\left(T, 0, h_{1}\right)=-\frac{M}{2} \frac{\partial^{2} f_{M}^{o}}{\partial h_{1}^{2}}\left(T, 0, h_{1}\right)=: \chi_{1,1}\left(T, h_{1}, M\right)
$$

that appears in the critical condition (3.11) is well known in the theory of surface critical phenomena [8, [7]. It takes the scaling form

$$
\chi_{1,1}\left(T, h_{1}, M\right) \sim \log |t|^{-1} \tilde{\mathcal{Q}}_{1}\left(h_{1}|t|^{-\Delta_{1}}, \frac{|t|^{-\nu}}{M}\right)+\tilde{\mathcal{Q}}_{2}\left(h_{1}|t|^{-\Delta_{1}}, \frac{|t|^{-\nu}}{M}\right)
$$

where $\Delta_{1}$ is the gap exponent corresponding to $h_{1}$ (see [9, 10, 11] for extensive treatments). In the limit $z=\left(|t|^{-\nu} / M\right) \rightarrow 0$, which corresponds to first taking the thermodynamic limit and then approaching the critical temperature, it diverges logarithmically:

$$
\chi_{1,1}(T) \sim \log |t|^{-1}
$$

This fact is closely related to the anomalous logarithmic divergence of the specific heat occuring in the two-dimensional Ising lattice. Consequently, at finite width and at the critical temperature $(z \rightarrow \infty)$, the FSS behaviour of $\chi_{1,1}$ at zero variational field is given by

$$
\chi_{1,1}\left(T_{c}, M\right) \sim \log M
$$

These results at hand, we can now return to the critical condition

$$
1 \sim \chi_{1,1}\left(T_{M}, 0, M\right) \sim \log t_{M}{ }^{-1} \tilde{\mathcal{Q}}_{1}\left(0, z_{M}\right)+\tilde{\mathcal{Q}}_{2}\left(0, z_{M}\right)
$$

Again, the cases $z_{M} \rightarrow$ const and $z_{M} \rightarrow \infty$ as $M \rightarrow \infty$ can be ruled out by (3.16) and (3.15), respectively. For $z_{M} \rightarrow 0$, (3.14) becomes $1 \sim \log t_{M}{ }^{-1}$, or $t_{M} \sim 1$, which is consistent with $z_{M} \rightarrow 0$. We therefore conclude that

$$
t_{M}=\left(T_{M}-T_{c}\right) / T_{c} \sim 1 \quad(M \rightarrow \infty)
$$

The critical temperatures of the sequence of test-systems based on open inhomogeneously magnetized strips do not converge to $T_{c}$ which conforms to our above observation. Note that this result is not merely an anomaly of the two-dimensional Ising lattice. It does not, as it might seem, depend on the logarithmic singularity of $\chi_{1,1}(T)$. A similar calculation for the case of a non-zero exponent $\gamma_{1,1}$ corresponding to $\chi_{1,1}$ gives just the same behavior for the $t_{M}$ 's. It has tacitly been assumed, though, that the edge itself cannot become critical at a temperature 
different from the bulk's $T_{c}$. This restricts the above argument to quasi-one-dimensional testsystems.

We can finally use these results to investigate the scaling of the mean-field susceptibilities (2.24) with strip width $M$ near the respective mean-field critical points $T_{M}$. Analogous to (3.5), we find the mean-field susceptibility

$$
\chi_{M}^{m f}(T)=\chi_{M}\left(T, h_{1}(T)\right)+J \frac{2}{M} \frac{\left(\chi_{1}\left(T, h_{1}(T)\right)\right)^{2}}{1-J \chi_{1,1}\left(T, h_{1}(T)\right)} .
$$

Here $\chi_{M}=\partial^{2} f / \partial H^{2}, \chi_{1}=-(2 / M) \partial^{2} f / \partial H \partial h_{1}$ and $\chi_{1,1}=-(2 / M) \partial^{2} f / \partial h_{1}{ }^{2}$ are the "bulk" susceptibility and the two "surface" susceptibilities well known in the standard treatment of surface critical phenomena. The mean-field susceptibility $\chi_{M}^{m f}(T)$ differs from the free susceptibility $\chi_{M}(T)$ of a strip of the same geometry in two ways: by the action of the variational boundary field $h_{1}(T)$ in the first term of the right hand side of (3.18), and by an explicit mean-field divergence in the second term.

Both of these contributions can be shown to become irrelevant in the limit of large $M$. In this limit, $\xi_{M}\left(T_{M}\right) / M \rightarrow 0$ in view of (3.17), so that the two surface susceptibilities $\chi_{1}$ and $\chi_{1,1}$ become independent of the strip width $M$. Setting them constant and expanding in small temperature differences above $T_{M}$, we arrive at

$$
\chi_{M}^{m f}(T) \sim \chi_{M}\left(T, h_{1}(T)\right)+\frac{\text { const }}{M} \frac{1}{T-T_{M}} .
$$

Thus, in the limit of large width $M$, the explicit mean-field contribution is suppressed by a factor $1 / M$. Furthermore, the surface field $h_{1}(T)$ appearing below $T_{M}$ does not affect thermodynamic properties of the bulk at large $M$, since the thermodynamic limit is independent of boundary conditions.

We thus encounter the paradoxical situation of a singularity in the "open" mean-field approximants which becomes spurious, as the limit $M \rightarrow \infty$ is taken. That is, even though thermodynamic functions exhibit a (suppressed) singularity at a temperature $T_{M}>T_{c}$, this singularity does not in the limit $M \rightarrow \infty$ correspond to a change in the system's thermodynamic properties. Evidently, no useful information can be drawn from these mean-field singularities, and any attempt to extrapolate to the underlying $2-d$ Ising lattice must fail.

The true thermodynamic singularity of the mean-field susceptibility develops right at $T_{c}$ in 
the first contribution to (3.18), by conventional reduction of the finite-size rounding of the bulk susceptibility $\chi_{M}$ as $M \rightarrow \infty$.

\section{Conclusions}

In the present paper a generalized mean-field theory based on the Peierls-Bogoliubov inequality is used to define quasi-one-dimensional approximants for the 2- $d$ Ising lattice. By convexity arguments, all but one of the minimization conditions for the varational parameters can be solved explicitly, a finding that is not restricted to the Ising model but holds generally for systems with a scalar order parameter. Thereby a systematic classification of PB approximants is possible. We singled out two types of strips: periodically "closed" strips with rotational symmetry in the direction transverse to the axis of infinite extent and "open" strips of inhomogeneous magnetization.

By standard finite-size scaling, the former are shown to display a coherent anomaly. Estimates of critical exponents of the underlying, fully interacting Ising system can be extracted. At this point, it has to be stressed that the variational method presented above should not be advocated as a new, superior numerical tool for computing transition temperatures or critical exponents. The estimates calculated above are clearly inferior to those obtained by Hu et al. [12]. In the original CAM scheme, based on ad hoc self-consistency equations, mean-field critical temperatures behave like $\left(T_{M}^{C A M}-T_{c}\right) \sim M^{-1 / \nu}$, whereas (3.7) states $\left(T_{M}^{P B}-T_{c}\right) \sim M^{-1 / \gamma}$. That is, the convergence of the PB critical temperatures is slower than in the scheme of $\mathrm{Hu}$ et al. (which in turn is slower than that of the conventional phenomenological renormalization group procedure [14]). In the PB scheme, the asymptotic regime of FSS power laws is reached only for very large strip width. Corrections to scaling are therefore expected to play an important rôle.

Nevertheless, we believe that our findings are of interest in their own right, since the appearance of a coherent anomaly in the family of "closed" approximants can be given a transparent interpretation as a FSS phenomenon.

In sharp contrast to expectations, the "open" strips, which are found to be the optimal family of approximants in the sense of the $\mathrm{PB}$ inequality, give rise to a spurious criticality at a temperature different from the $2-d$ Ising critical temperature. Any extrapolation to the full 
two-dimensional system based on the mean-field critical behaviour of this family must therefore fail. This unexpected result clearly shows that variational descriptions of many particle systems should be used with utmost caution.

\section{Acknowledgements}

It is a pleasure to thank Heinz Horner for many stimulating discussions and Jochen Rau for the careful reading of the manuscript.

\section{References}

[1] Burley D M (1972), in: Phase Transitions and Critical Phenomena, Vol. 2, C Domb and M Green (eds.) Academic Press, London

[2] Bethe H A (1935) Proc. Roy. Soc. A 150552

[3] Peierls R (1934) Phys. Rev. 54918

[4] Trudeau Y and M L Plumer, preprint, October 1994

[5] Suzuki M (1986) J. Phys. Soc. Japan 554205

[6] Suzuki M, M Katori and X Hu (1987) J. Phys. Soc. Japan 563092

[7] Barber M N (1983) in: Phase Transitions and Critical Phenomena, Vol. 8, C Domb and J L Lebowitz (eds.) Academic Press, London

[8] Binder K (1983) in: Phase Transitions and Critical Phenomena, Vol. 8, C Domb and J L Lebowitz (eds.) Academic Press, London

[9] Au-Yang H and M E Fisher (1975) Phys. Rev. B 113469

[10] Au-Yang H and M E Fisher (1980) Phys. Rev. B 213956

[11] McCoy B M and T T Wu (1967) Phys. Rev. 162436

[12] Hu X, M Katori and M Suzuki (1987) J. Phys. Soc. Japan 563865

[13] Henkel M and G Schütz (1988) J. Phys. A 212617 
[14] Nightingale P (1976) Physica 83 A 56

\section{Figure Captions}

Fig. 1: Mean-field critical temperature $T_{M}$ of closed approximants as a function of inverse strip width $1 / M$.

Fig. 2: Estimate of the susceptibility exponent derived from the ratio of prefactors $\bar{\chi}_{M}$ of the mean-field susceptibility for two successive strip widths $M$ and $M^{\prime}=M+1$ as a function of $2 /\left(M+M^{\prime}\right)$.

Fig. 3: Mean-field critical temperature $T_{M}$ of open approximants as a function of inverse strip width $1 / M$. Note that they do not extrapolate to $T_{c}$, as $M \rightarrow \infty$. 

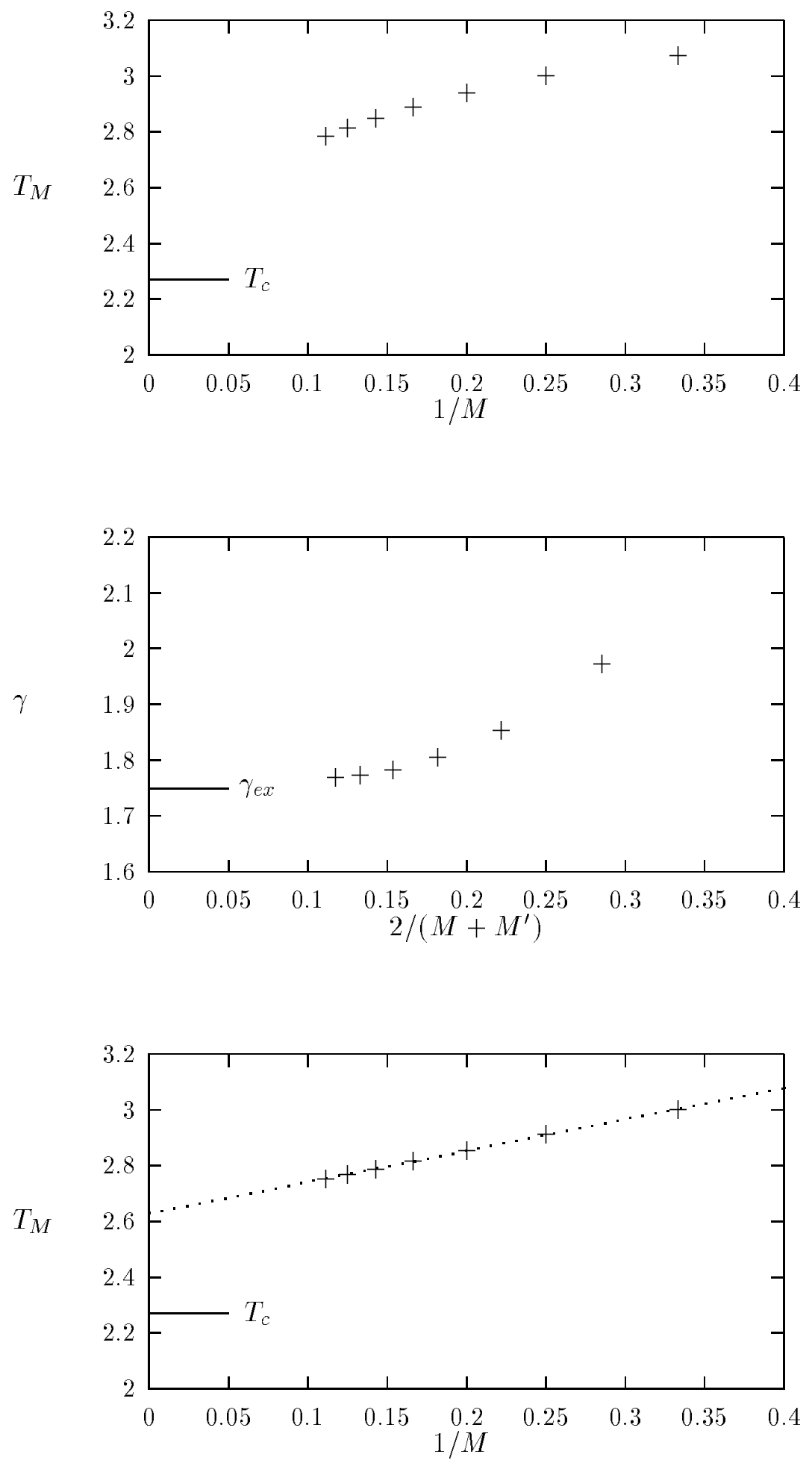